\title{
Expression of stem cell markers nanog and PSCA in gastric cancer and its significance
}

\author{
XUANZHONG ZHAO, FENG WANG and MINGXING HOU \\ Department of General Surgery, Affiliated Hospital of Inner Mongolia Medical University, \\ Hohhot, Inner Mongolia 010059, P.R. China
}

Received October 20, 2014; Accepted July 16, 2015

DOI: $10.3892 / \mathrm{ol} .2015 .3884$

\begin{abstract}
The present study aimed to determine the expression of stem cell markers Nanog compared with PSCA in gastric cancer tissues and adjacent normal tissues, and to investigate the association between tumor stem cells and initiation, progression, metastasis, and prognosis of gastric cancer. One hundred chemotherapy- and radiotherapy-naive patients with pathologically confirmed gastric cancer were enrolled from the General Surgery Department and Surgical Oncology Department of the Affiliated Hospital of Inner Mongolia Medical University (Hohhot, P.R. China), between October 2011 and June 2013. Surgically resected specimens of cancer tissues and adjacent normal tissues $(>5 \mathrm{~cm}$ from the boundary of cancerous component) were collected. The mRNA expression levels of Nanog and PSCA in those tissues was determined by reverse transcription-quantitative polymerase chain reaction (RT-qPCR). The correlation between the expression of stem cell markers Nanog and PSCA in gastric cancer tissues and clinicopathological factors was analyzed. The qPCR results demonstrated that the relative expression of Nanog was increased in gastric cancer tissues compared with in the adjacent tissues $(\mathrm{P}<0.05)$; and relative expression of PSCA was reduced in gastric cancer tissues compared with adjacent tissues $(\mathrm{P}<0.05)$. The expression of Nanog and PSCA in gastric cancer tissues was associated with tumor differentiation. The expression of Nanog was increased in poorly-differentiated and undifferentiated tumors compared with moderately- and well-differentiated tumors $(\mathrm{P}<0.05)$. The expression of PSCA was reduced in poorly differentiated and undifferentiated tumors compared with moderately- and well-differentiated tumors $(\mathrm{P}<0.05)$. However, the expression of Nanog and PSCA was not associated with age, gender, tumor size, TNM stage, depth of invasion, or lymph node metastasis. Therefore, Nanog and
\end{abstract}

Correspondence to: Professor Mingxing Hou, Department of General Surgery, Affiliated Hospital of Inner Mongolia Medical University, 1 Tongdaobei Street, Hohhot, Inner Mongolia 010059, P.R. China

E-mail: houmingxingnmg@163.com

Key words: gastric cancer, nanog, PSCA, RT-qPCR
PSCA may have potential as molecular markers to reflect the differentiation status of gastric cancer.

\section{Introduction}

Gastric cancer is the most common malignant tumor of the digestive system in the world, and is the most common malignant tumor in China (1). According to the cancer control program of the World Health Organization, 7 million patients died of cancer worldwide each year, and 700,000 of them died of gastric cancer (2). Moreover, 934,000 new cases of gastric cancer occur worldwide each year, $70 \%$ of them occurred in developing countries, half of them occurred in eastern Asia, and 42\% ( 400,000) of them occurred in China. The incidence and mortality of gastric cancer in China are $>2$ times the worldwide average $(1,2)$.

Previous studies have demonstrated that location of gastric cancer is associated with clinicopathological features. The clinicopathological features of gastric cancer in the cardia, the upper $1 / 3$ stomach, and the distal stomach are quite independent from one other (3). The clinicopathological features of gastric body cancer and distal gastric cancer are similar in age of onset, tumor size and other features. The clinicopathological features of gastric body cancer are in between those of the proximal gastric cancer and distal gastric cancer (2). In recent decades, the incidence of distal gastric cancer has been significantly reduced, while that of proximal gastric cancer (cardia adenocarcinoma) significantly increased in residents of European and American countries (4,5). Gastric cancer also varies by gender, age, ethnicity, and region (6). The incidence of gastric cancer is higher among men compared with women (7). The male/female incidence ratio of gastric cancer is increased in rural areas $(20.93 / 100,000$ in men and $10.6 / 100,000$ in women) compared with in urban areas (1). The incidence is significantly higher in eastern coastal and northwest regions compared with southern China (8). The incidence of gastric cancer is higher in the elderly compared with the young, with a trend for an increasingly higher incidence and poor prognosis in the young (9). It has been reported that age may be a major factor affecting early diagnosis of gastric cancer. Since early clinical symptoms are similar to those of gastritis and gastric ulcer, young patients may be discharged following treatment, leading to delayed diagnosis and treatment for gastric cancer. 
Despite a large number of studies on the etiology and pathogenesis of gastric cancer, these issues have not been fully elucidated $(10,11)$. There are numerous risk factors for gastric cancer, including Helicobacter pylori (HP) infection, genetic factors, environmental factors, dietary factors, history of gastric disorders, and psychological factors (12-14). There are various treatment methods for gastric cancer, including surgery, chemotherapy, radiotherapy, and immunotherapy. Numerous factors influence the survival rate of gastric cancer, including the stage of disease, surgical treatment, tumor size, tumor location, histological type, and particularly the degree of differentiation (15), pathological stage, and lymph node metastasis (16). At present, the 5-year survival rate of middle and late stage gastric cancer is $\sim 20 \%$, with a trend for increased mortality in the young $(2,17)$. Tumor metastasis is the most notable cause of mortality in patients with gastric cancer. Investigating risk factors and invasion and metastasis characteristics of gastric cancer, and providing early intervention and standardized and effective combination therapy may reduce the incidence of gastric cancer, increase survival, and significantly improve prognosis $(18,19)$. Investigating molecular pathogenesis of gastric cancer may aid in the identification of genes, mechanisms and other specific indicators associated with the pathogenesis of gastric cancer at the molecular level. These findings may have important theoretical significance and clinical practical value for the diagnosis and treatment of gastric cancer and provide guidance for improving the quality of life of patients.

Stem cells are a class of cells that possess self-renewal, proliferation and differentiation potential. They are often classified into embryonic stem cells (ESCs) and adult stem cells according to the stage of development (20). With recent progress in the research of stem cells and tumor cells, there is evidence that the process of tumorigenesis may be associated with stem cells (20). Tumorigenic cells and stem cells share similar characteristics in terms of their biology and growth control mechanisms. Rare cancer cells exist that act in a similar way to stem cells during tumor formation. Certain cancer cells have the potential for self-renewal and differentiation, and appear to be the root cause of tumor proliferation, growth, invasion, metastasis, and recurrence, and thus are called tumor stem cells (TSCs) (20). TSCs maintain the viability of tumor cell populations through self-renewal and unlimited proliferation. Moreover, their movement and migration capabilities provide the potential for metastasis of the tumor cells. TSCs may be senescent for a long period and contain numerous drug-resistant molecules, and thus are less susceptible to various external physical and chemical factors (21).

In 2008, Mani et al (22) reported that epithelial/mesenchymal transition (EMT) induced a proportion of tumor cells to exhibit TSC-like characteristics. This finding indicted that TSCs may be the basis of tumor invasion and metastasis, promoting tumor metastasis and proliferation by acquiring certain features of mesenchymal cells. EMT occurs in malignant tumors: The process of EMT involves epithelial cells acquiring the features of mesenchymal cells under certain physiological and pathological circumstances, and then leaving the primary tumor, breaking through the vessel wall, entering the blood circulatory system, invading surrounding tissues and metastasizing to distant sites, which often leads to a poor prognosis. It has been demonstrated that TSCs are closely associated with EMT. The majority of circulating tumor cells have characteristics of EMT and stem cells (23). Invasion, metastasis and drug resistance are the major causes of treatment failure in gastric cancer (24). The survival of patients with gastric cancer may be greatly improved by detection and targeted killing of circulating TSCs.

Bonnet et al (25) first reported the presence of leukemic stem cells specifically expressing the CD34 $/$ CD $38^{-}$phenotype in acute myelogenous leukemia in 1997. In recent years, specific surface markers for TSCs have been successfully identified and isolated in solid tumors, including breast cancer, brain cancer, prostate cancer, ovarian cancer, melanoma, and colon cancer (26-28), providing strong support for the TSC theory. However, surface marker specific to TSCs in gastric cancer have not yet been determined.

ESCs are a class of pluripotent stem cells derived from cells within the blastocyst. They have an in vitro ability of unlimited proliferation, self-renewal and multilineage differentiation, and may be induced to differentiate into almost all tissues and organs in vitro and in vivo. Major regulatory factors for the maintainenance of undifferentiated ESCs are OCT3/4, SOX2 and homologous protein transcription factor Nanog. The signaling pathways that maintain stemness in ESCs have been demonstrated to include LIF/gp130-Jak-STAT3, Wnt, bone morphogenetic protein, fibroblast growth factor and transforming growth factor (TGF) $\beta$-activin/nodal signaling pathways (29). Nanog is key in maintaining the pluripotency of ESCs and may serve a role independent of the LIF signal. It selectively inhibits the expression of differentiation genes or promotes the expression of pluripotent genes by binding to regulatory regions of target genes.

In 2003, Wang et al (30) demonstrated that an ENK gene specifically expressed in ESCs during screening of the mouse cDNA library, however its function was unknown. Subsequently, Mitsui (31) and Chambers et al (32) demonstrated that this gene promoted self-proliferation and multilineage differentiation, and named it Nanog. Nanog is a transcription factor expressed in primordial germ cells, embryonal carcinoma cells, testicular carcinoma in situ, breast cancer, and gliomas (33). As an important member of the Antennapedia (Antp)-class neurokinin (NK) family, Nanog is a homeobox gene located in the 12 p13 region of human chromosome 12 . Its cDNA consists of 2184 nucleotides, encoding a protein of 305 amino acids, which is comprised of 3 domains: An $\mathrm{N}$-terminal domain containing 96 serine- and threonine-rich residues; a C-terminal domain containing obvious $\mathrm{W}$ repeat sequences; and a homology domain that interacts with proteins and binds to DNA.

Nanog is an important transcription factor for maintaining the self-renewal and pluripotency of ESCs. It regulates the fate of inner cell mass of blastocysts in the embryo during early development. During late development, it maintains the pluripotency of the ectoderm by controlling the activity of transcription factors associated with differentiation; however, as development continues, the expression of Nanog begins to reduce. Mitsui et al (31) investigated different stages of embryonic development in mice and demonstrated that the ectoderm did not develop in a Nanog-deficient embryo. In adult tissues, Hart et al (34) detected low expression of Nanog in the testis, 
ovary, liver, spleen, and kidney, and weak expression in the thymus, brain, intestine, uterus, and heart by fluorescent immunohistological analysis.

Nanog interacts with SOX2 and OCT4 in the regulation of gene function, including its own negative feedback regulation. Nanog, SOX2, and OCT4 expression are important in early embryonic development, mainly by regulating the TGF- $\beta$ and Wnt signaling pathways (35). These molecules serve key regulatory roles in the in vitro proliferation and pluripotency of ESCs. They bind to the promoter of their target genes and cooperate to form a regulation loop in an interdependent manner, therefore inducing their own encoding genes and genes associated with signal transduction pathways and regulating the gene transcription in ESCs (35). These 3 transcription factors, Nanog, SOX2, and OCT4, are at the core of the transcriptional regulation of both mouse and human ESCs: ESC differentiation occurs when expression of one of the 3 regulatory factors is lost $(35,36)$. SOX 2 is a high-mobility non-histone box domain protein, and Nanog and OCT4 are homeobox domain proteins; SOX2 stabilizes the pluripotency of ESCs by maintaining the appropriate expression levels of OCT4, while Nanog and OCT4 maintain the pluripotency primarily by blocking the differentiation of ESCs. Oct4 is a member of the family of the POU transcription factor family, and its expression is associated with the functions of germ cells and ESCs (35). Nanog-deficient ESCs have a reduced potential for multilineage differentiation. OCT4-deficient ESCs still express Nanog, however Nanog expression alone is insufficient to maintain the undifferentiated state of ESCs. Therefore, Nanog and OCT4 are functionally associated (35).

In addition to being expressed in ESCs, Nanog is also expressed in germ cell tumor cells, somatic cell tumor cells and other tumor cells. A study investigating Nanog expression in tumor tissues and in normal testis demonstrated that Nanog was highly expressed in testicular carcinoma in situ, seminoma, and embryonal carcinoma, whereas not expressed in normal testis, indicating that high levels of Nanog expression may be a germ cell tumor marker (37). Nanog was also demonstrated to be abnormally highly expressed in bladder, breast, glioma and lung cancer tumors; to be associated with tumor size, malignancy, and clinical prognosis; and nanog-expressing cells exhibit features of ESCs in high-grade tumors (38). Ye et al (39) examined the association between Nanog expression and the development of cervical cancer using molecular biology techniques, and demonstrated that Nanog expression levels were reduced in cervical squamous cell carcinoma, cervical intraepithelial neoplasia (CIN) compared with normal cervical epithelium. In addition the authors demonstrated that a close association existed between reduced Nanog expression and increased size of cervical squamous cell carcinoma. Siu et al (40) examined the expression of Nanog in gestational trophoblastic disease, and observed abnormally high expression of Nanog in hydatidiform mole and choriocarcinoma tissues, and that knockout of the Nanog gene in choriocarcinoma cell line JEG-3 cells using siRNA induced apoptosis and inhibited the invasiveness of those tumor cells. Chen et al (41) examined the expression of Nanog in tumor tissues and adjacent tissues in patients with gastric cancer by qPCR and its association with clinicopathological parameters. It was demonstrated that the mRNA expression levels of Nanog were increased in the tumor tissues compared with adjacent tissues, and that positive expression of Nanog was associated with tumor differentiation, indicating that Nanog could be used as novel diagnostic molecular marker for the differentiation status of gastric cancer (41).

In 1998, while examining prostate cancer gene expression in LAPC-4 mice (a mouse model of prostate cancer), Reiter et al (42) identified a tumor antigen associated with prostate cancer that exhibited a $30 \%$ homology with stem cell antigen-2 (SCA-2) by specific difference analysis, which was therefore termed prostate stem cell antigen (PSCA). PSCA is a glycosylphosphatidylinositol (GPI)-anchored protein expressed in prostate epithelial cell surface, and is a member of the Thy-1/Ly- 6 family. The human PSCA gene is located on chromosome 8q24.2 and involved in various functions. The cDNA consists of $660 \mathrm{bp}$ and encodes a protein of 123 amino acids, which contains highly conserved cysteine residues characteristic of the gene family, an N-terminal signal sequence, and a C-terminal GPI anchor sequence that anchors it to the cell surface, and $4 \mathrm{~N}$-glycosylation sites. Proteins of the Thy- 1 family are associated with activation and proliferation of $\mathrm{T}$ cells and survival of stem cells, and proteins of the Ly- 6 family are associated with tumorigenesis. The majority of GPI-anchored proteins similar to PSCA are associated with activation of T cells.

PSCA is highly expressed in tissues, such as the bone, liver and lymph nodes, in the majority of patients with prostate cancer and patients with metastases, while low levels or no expression was observed in other normal tissues, including urinary epithelium, benign prostatic hyperplasia, normal prostate epithelium, and non-prostate tissues such as the small intestine and kidney (43). These observations determine the significance of measuring PSCA in guiding early diagnosis, detection of metastasis, and determination of prognosis for prostate cancer. Ono et al (44) identified reduced expression of PSCA in esophageal, gastric and gallbladder cancer, indicating that different functions of PSCA are associated with tissue types and the state of the cells themselves (45).

It has been indicated that single nucleotide polymorphisms (SNPs) of the PSCA gene, rs2976392-A allele and rs2294008-T allele, are closely associated with diffuse gastric cancer in the population of Japan and South Korea (46), in addition to the enterotype and diffuse gastric cancer in the Chinese population (47). Qiao et al (48), also demonstrated that the PSCA gene polymorphisms were associated with susceptibility to diffuse gastric cancer. An in vitro study identified that the number of colonies formed from the gastric cancer cell line HSC57 stably transfected with PSCA cDNA was significantly increased compared with that from the cell line not expressing PSCA, that PSCA mRNA was highly expressed in normal gastric mucosa, mainly in isthmus of gastric glands, and that PSCA mRNA expression was not detected in gastric cancer tissues (49). These findings indicated that PSCA may inhibit gastric cancer cell proliferation and that the PSCA rs2294008-T allele may reduce the inhibitory effect of PSCA on gastric epithelial cell proliferation by 
downregulating the PSCA expression, thereby increasing the risk of gastric cancer.

\section{Patients and methods}

Experimental setup. Paired samples of gastric cancer tissues and normal adjacent tissues were collected from 100 patients with pathologically confirmed gastric cancer who were enrolled from General Surgery Department and Surgical Oncology Department of Affiliated Hospital of Inner Mongolia Medical University (Hohhot, China) between October 2011 and June 2013. All patients treated were fully informed of the treatment methods, characteristics, the complications that may arise, and signed informed consent was obtained. This study was approved by The Ethics Committee of the Affiliated Hospital of Inner Mongolia Medical University (no. 20130423).

Data regarding the patients' gender, age, surgical treatment, pathology, laboratory tests, and preoperative treatment was collected. The 100 cases included 74 males and 26 females,

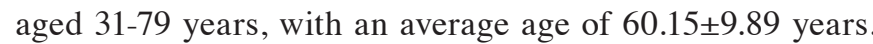
In terms of tumor differentiation, 37 patients had well- and moderately-differentiated cancers and 63 had poorly-differentiated and undifferentiated cancers. Adjacent tissues were normal gastric wall tissues $5 \mathrm{~cm}$ from the tumor lesions and were resected in the same surgery as the cancer tissues. All apparatus were immersed in DEPC water to remove RNA degrading enzymes prior to use. The specimens were placed in cryogenic vials under liquid nitrogen within 5 min of resection, and then transferred to a $-80^{\circ} \mathrm{C}$ freezer. All pathological specimens were confirmed by two physicians of the pathology department.

RNA extraction procedures and cDNA first strand synthesis. Total RNA was extracted from the 100 patient tumor samples and adjacent non-cancerous tissues, using an RNeasy plus universal Mini kit (Qiagen Co. Ltd. Shanghai, China) according to the manufacturer's instructions. The extracted RNA was stored at $-80^{\circ} \mathrm{C}$. The total RNA concentration and purity was determined using a NanoDrop 2000C (Thermo Fisher Scientific Inc., Tokyo, Japan). The RNA integrity was determined by electrophoresis through a $2 \%$ agarose, $1.5 \%$ ethidium bromide gel. The samples were run at $120 \mathrm{~V}$ for approximately $20 \mathrm{~min}$. The electrophoresis gel plate was observed under an automatic gel imager (BG-GDSAUTO, Baijing Biotechnology Co. Ltd. Beijing, China) and photographed. The clear presence of 18 and 28s peaks indicated suitability for reverse transcription. cDNA was synthesized using a TIANScript cDNA First Strand Synthesis kit (Tiangen Co. Ltd. China) according to the manufacturer's instructions.

qPCR detection. Primers were designed according to the complete sequences of Nanog, PSCA, and GAPDH provided by PubMed, and synthesized by Invitrogen (Shanghai, China) and are presented in Table I. qPCR was performed using a QuantiFast SYBR Green PCR Kit according to the manufacturer's instructions. The reaction mixture contained $2 \mu \mathrm{l}$ each of upstream and downstream primers (Table I), $10 \mu \mathrm{l}$ of 2x QuantiFast SYBR Green PCR Master Mix and $2 \mu \mathrm{l}$ of template cDNA, and RNase-free water to a final volume
Table I. Primers for Nanog, PSCA, and GAPDH amplification.

\begin{tabular}{lc}
\hline Primer name & \multicolumn{2}{c}{ Primer sequence } \\
\hline Nanog & F: 5'-ACCCAGCTGTGTGTACTCAA-3' \\
& R: 5'-GGAAGAGTAAAGGCTGGGGT-3' \\
PSCA & F: 5'-CCCAGCATTCTCCACCCTTA-3' \\
& R: 5'-TCATCTCAGCCTTTACCGGG-3' \\
GAPDH & F: 5'-CCTCAACGACCACTTTGTCA-3' \\
& R: 5'-TTACTCCTTGGAGGCCATGT-3'
\end{tabular}

Table II. Relative Nanog and PSCA mRNA expression in cancer tissues and adjacent tissues.

\begin{tabular}{lcccc}
\hline Tissue & $\begin{array}{c}\text { Nanog } \\
\text { mRNA }\end{array}$ & P-value & $\begin{array}{c}\text { PSCA } \\
\text { mRNA }\end{array}$ & P-value \\
\hline Cancer tissue & $1.24 \pm 0.47$ & & $0.36 \pm 0.30$ & \\
Adjacent tissue & $1.01 \pm 0.02$ & 0.000 & $1.00 \pm 0.01$ & 0.000 \\
\hline
\end{tabular}

of $20 \mu \mathrm{l}$. The mixture was preheated at $95^{\circ} \mathrm{C}$ for $5 \mathrm{~min}$ to avoid interference of primer dimers, followed by 40 cycles of denaturation at $95^{\circ} \mathrm{C}$ for $10 \mathrm{sec}$ and annealing/extension at $60^{\circ} \mathrm{C}$ for $30 \mathrm{sec}$, on a Veriti 96 well thermocycler (Applied Biosystems Life Technologies, Foster City, CA, USA). Curves were calculated and plotted using the software provided by the using the Real-time PCR system (7500) (Applied Biosystems, China). Ct represents the number of amplification cycles performed when the fluorescence signal of PCR product reached the established threshold. Relative mRNA expression of Nanog and PSCA genes was assessed using the $2^{-\Delta \Delta \mathrm{Ct}}$ method.

Statistical analysis. Each sample was measured 3 times. Relative expression in cancer tissues and adjacent normal tissues in patients with gastric cancer, and correlation between expression of Nanog and PSCA and clinicopathological parameters were analyzed using SPSS software, version 13.0 (SPSS, Inc., Chicago, IL, USA) using $t$-test and non-parametric rank sum test. $\mathrm{P}<0.05$ was considered to indicate a statistically significant difference.

\section{Results}

Determination of total RNA integrity and purity and $q P C R$. Total RNA extracted from gastric cancer tissues was observed following agarose gel electrophoresis. 28s rRNA and 18s rRNA bands were clearly visible and $5 \mathrm{~s}$ rRNA bands were not distinct, with OD values (A260/A280) measured to be 1.8-2.0, indicating almost no protein contamination occurred and a high purity of total RNA. qPCR curves, amplification plots and melting curves of Nanog, PSCA and GAPDH genes were generated using the software of the qPCR analyzer. Amplification curves of each sample reached a plateau. Melting curves demonstrated no evidence of primer dimer signals or other unspecific fluorescence signals. 
Table III. Correlation between relative Nanog and PSCA mRNA expression and clinicopathological parameters in gastric cancer tissues.

\begin{tabular}{|c|c|c|c|c|c|}
\hline Clinicopathological parameters & $\mathrm{n}$ & Nanog mRNA & P-value & PSCA mRNA & P-value \\
\hline \multicolumn{6}{|l|}{ Age } \\
\hline$\leq 60$ & 50 & $1.26 \pm 0.46$ & & $0.38 \pm 0.32$ & \\
\hline$>60$ & 50 & $1.24 \pm 0.45$ & 0.824 & $0.33 \pm 0.27$ & 0.833 \\
\hline \multicolumn{6}{|l|}{ Gender } \\
\hline Male & 74 & $1.27 \pm 0.45$ & & $0.36 \pm 0.30$ & \\
\hline Female & 26 & $1.15 \pm 0.54$ & 0.310 & $0.35 \pm 0.28$ & 0.878 \\
\hline \multicolumn{6}{|l|}{ Size of tumor } \\
\hline$\leq 5$ & 52 & $1.23 \pm 0.48$ & & $0.36 \pm 0.30$ & \\
\hline$>5$ & 48 & $1.24 \pm 0.47$ & 0.899 & $0.35 \pm 0.29$ & 0.997 \\
\hline \multicolumn{6}{|l|}{ TNM stage } \\
\hline $\mathrm{I}+\mathrm{II}$ & 35 & $1.19 \pm 0.54$ & & $0.35 \pm 0.28$ & \\
\hline $\mathrm{III}+\mathrm{IV}$ & 65 & $1.26 \pm 0.44$ & 0.484 & $0.36 \pm 0.30$ & 0.990 \\
\hline \multicolumn{6}{|l|}{ Degree of differentiation } \\
\hline $\begin{array}{l}\text { Moderately- and } \\
\text { well-differentiated }\end{array}$ & 37 & $1.10 \pm 0.28$ & & $0.54 \pm 0.25$ & \\
\hline $\begin{array}{l}\text { Poorly-differentiated } \\
\text { and undifferentiated }\end{array}$ & 63 & $1.32 \pm 0.54$ & 0.000 & $0.25 \pm 0.27$ & $<0.001$ \\
\hline \multicolumn{6}{|l|}{ Depth of invasion } \\
\hline $\mathrm{T} 1, \mathrm{~T} 2$ & 19 & $1.33 \pm 0.44$ & & $0.42 \pm 0.28$ & \\
\hline $\mathrm{T} 3, \mathrm{~T} 4$ & 81 & $1.22 \pm 0.48$ & 0.337 & $0.34 \pm 0.30$ & 0.122 \\
\hline \multicolumn{6}{|l|}{ Lymph node metastasis } \\
\hline Yes & 75 & $1.24 \pm 0.46$ & & $0.35 \pm 0.30$ & \\
\hline No & 25 & $1.24 \pm 0.51$ & 0.979 & $0.36 \pm 0.28$ & 0.950 \\
\hline
\end{tabular}

$\mathrm{P}<0.05$ for comparison between groups.

qPCR detection of nanog and PSCA mRNA expression in gastric cancer tissues and adjacent tissues. With GAPDH as a reference gene, the relative expression of Nanog was $1.24 \pm 0.47$ in cancer tissues and $1.01 \pm 0.02$ in adjacent tissues in the 100 patients with gastric cancer, as revealed by qPCR analysis. The relative expression of Nanog was significantly increased in gastric cancer tissues compared with the corresponding adjacent tissues $(\mathrm{P}<0.01)$. The relative mRNA expression levels of PSCA were $0.36 \pm 0.30$ in cancer tissues and $1.00 \pm 0.01$ in adjacent tissues. The relative expression of PSCA was significantly reduced in gastric cancer tissues compared with the corresponding adjacent tissues $(\mathrm{P}<0.01)$. The results are presented in Table II.

Correlation between nanog and PSCA mRNA expression and clinicopathological parameters in cancer tissues. As revealed by statistical analysis, the relative expression of Nanog was significantly increased in poorly-differentiated and undifferentiated cancer tissues $(1.32 \pm 0.54)$ compared with moderately- and well-differentiated cancer tissues $(1.10 \pm 0.28)(\mathrm{P}<0.01)$; and the relative expression of PSCA was significantly reduced in poorly-differentiated and undifferentiated cancer tissues $(0.25 \pm 0.27)$ compared with moderately- and well-differentiated cancer tissues $(0.54 \pm 0.25)(\mathrm{P}<0.01)$. The results are presented in Table III.

\section{Discussion}

Gastric cancer is the most common malignant tumor of the digestive system, with an overall poor prognosis. The process from normal gastric mucosa, chronic superficial gastritis, chronic atrophic gastritis, intestinal metaplasia, dysplasia, to gastric cancer is characterized by the presence of differentially expressed genes. Early detection of genes associated with the development of gastric cancers and markers is important for the prevention of gastric cancer and early intervention in tumorigenesis, in addition to the subsequent prevention of malignancy development. It may provide the basis for prediction of gastric cancer, efficacy analysis, and development of prevention measures, and therefore more accurately guide combination of clinical targeted therapy, immunotherapy, and gene therapy, allowing individualized targeted treatment.

Nanog is an important transcription factor for maintaining the self-renewal and multilineage differentiation capacity of ESCs. It is also a commonly used stem cell marker. Although previous studies have demonstrated that Nanog is important in maintaining and regulating the self-renewal and multilineage differentiation capacity of stem cells, the exact underlying mechanism is complicated. The 5 ' terminal promoter of Nanog has been demonstrated to contain a composite Oct4/Sox 2 motif-binding site, which initiates and regulates the expression of Nanog (50). 
It has been demonstrated that Nanog and Oct- 4 interact with one another in regulating the self-renewal and multilineage differentiation capacity of stem cells, but the expression of Nanog involves many other factors. One study demonstrated that the most common cancer-associated gene, P53, binds to the Nanog promoter both in vivo and in vitro, and negatively regulates the expression of Nanog (51). FoxD3 is a transcription factor of the forkhead family, and is also an important factor that regulates Nanog gene expression (52).

PSCA is a biological marker for diagnosis and prognosis of prostate cancer and other tumors and may also serve as a candidate target for cancer therapy. Increased expression of $c$-myc oncogene was observed in $71 \%$ of patients with prostate cancer that demonstrated increased expression of PSCA (40). Both PSCA and $c$-myc are located on chromosome $8 \mathrm{q} 24$. Chromosome amplification is often observed in this region during human carcinogenesis. Therefore, increased expression of PSCA may also be observed in other tumors. However, PSCA serves an inhibitory role in esophageal and gastric cancers. PSCA promotes or suppresses tumorigenesis, depending on the tissue types and pathophysiological conditions, and therefore the mechanism requires further study.

In the present study, the expression of transcription factors Nanog and PSCA in gastric cancer and adjacent normal tissues was examined by qPCR, in order to investigate whether expression of Nanog and PSCA is involved in gastric cancer. Abnormally high expression of Nanog and low expression of PSCA were observed in gastric cancer tissues. These results indicate that Nanog and PSCA may be involved in the initiation, development and differentiation of gastric cancer and may serve as potential molecular markers of gastric cancer.

Nanog expression was significantly increased in gastric cancer tissues compared with adjacent tissues in the present study, indicating that high expression of Nanog may be associated with the pathogenesis of gastric cancer. PSCA expression was significantly reduced in gastric cancer tissues compared with adjacent tissues, indicating that downregulation of PSCA expression may be associated with gastric cancer tissue type and the differentiation state of the cells themselves. These findings also provide evidence for the theory of TSC.

In the present study the expression levels of Nanog and PSCA in gastric cancer tissues were associated with the degree of differentiation of gastric cancer, however no significant correlation between Nanog and PSCA expression and clinicopathological parameters, such as age, gender, tumor size, TNM stage, invasion depth, and lymph node metastasis., was observed. Therefore, Nanog and PSCA may have potential as molecular markers to reflect the differentiation status of gastic cancer.

\section{References}

1. Yang L: Incidence and mortality of gastric cancer in China World J Gastroenterol 12: 17-20, 2006.

2. Ferlay J, Shine HR, Bray F, Forman D, Mathers C and Parkin DM: Estimates of worldwide burden of cancer in 2008: GLOBOCAN 2008. Int J Cancer 127: 2893-2917, 2010.

3. Liu X, Liang H, Deng J, et al: Different parts of the patients with gastric cancer radical surgery clinical pathological characteristics and prognosis. J Chin Gastrointest Surg 13: 181-184, 2010.

4. Keeney $S$ and Bauer TL: Epidemiology of adenocarcinoma of the esophagogastric junction. Surg Oncol Clin N Am 15: 687-696, 2006.
5. Crane SJ, Richard Locke G III, Harmsen WS, Diehl NN, Zinsmeister AR, Joseph Melton L III, Romero Y and Talley NJ: The changing incidence Of oesophageal and gastric adenocarcinoma by anatomic sub-site. Aliment Phamacol Ther 25: 447-453, 2007.

6. Xu B and Wang J: Gastric cancer epidemiological studies. Chin J Cancer Prev Treat 1: 1-7, 2006.

7. Zou X, Sun X, Chen W, et al: gastric cancer and death of Chinese from 2003 to 2007. Tumor 32: 109-114, 2012.

8. Xu GW and Si JH. The stomach and duodenum diseases. In: Surgery. People's Health Publishing House, Beijing, pp1440-1467, 2008 (In Chinese).

9. Sun XD, Mu R, Zhou YS, Dai XD, Zhang SW, Huangfu XM, Sun J, Li LD, Lu FZ and Qiao YL: Analysis of mortality rate of stomach cancer and its trend in twenty years in China. Zhonghua Zhong Liu Za Zhi 26: 4-9, 2004 (In Chinese).

10. Bornschein J, Rokkas T, Selgrad M and Malfertheiner P: Gastric cancer: Clinical aspects, epidemiology and molecular background. Helicobacter 16 (Suppl 1): S45-S52, 2011.

11. Correia M, Machado JC and Ristimäki A: Basic aspects of gastric cancer. Helicobacter 14 (Suppl 1): S36-S40, 2009.

12. Li FP and Montesano R: Interactions of cancer susceptibility genes and environmental carcinogens. American Association for Cancer Research (AACR)-International Agency for Reserch on Cancer (IARC) Joint Conference. Cancer Res 54: 4243-4247, 1994.

13. Jakszyn P and Gonzalez CA: Nitrosamine and related food intake and gastric and oesophageal cancer risk: A systematic review of the epidemiological evidence. World J Gastroenterol 12: 4296-4303, 2006.

14. Kreuzer M, Straif K, Marsh JW, et al: Occupational dust and radiation exposure and mortality from stomach cancer among German uranium miners, 1946-2003. Occup Environ Med 69: 217-223, 2012.

15. Nakamura K, Ueyama T, Yao T, et al: Pathology and prognosis of gastric carcinoma. Findings in 10,000 patients who underwent primary gastrectomy. Cancer 70: 1030-1037, 1992.

16. Adachi Y, Yasuda K, Inomata M, et al: Pathology and prognosis of gastric carcinoma: Well versus poorly differentiated type. Cancer 89: 1418-1424, 2000.

17. Kamangar F, Dores GM and Anderson WF: Patterns of cancer incidence, mortality, and prevalence across five continents: Defining priorities to reduce cancer disparities in different geographic regions of the world. J Clin Oncol 24: 2137-2150, 2006.

18. He Y, Wang J, Zhao Z, Zheng $\mathrm{K}$ and Cui Y: EUS and comparative study of spiral CT TNM staging of gastric surgery. Zhong Guo Xiao Hua Nei Jing 2: 21-24, 2008 (In Chinese).

19. Yang Q, Zhu Z and Yutaka Y: Clinical and pathological features of early gastric cancer with distant metastasis. Zhong Guo Shi Yong Wai Ke Za Zhi 11: 873-875, 2007 (In Chinese).

20. Reya T, Morrison SJ, Clarke MF and Weissman IL: Stem cells, cancer and cancer stem cells. Nature 414: 105-111, 2001.

21. Dean M, Fojo T and Bates S: Tumour stem cells and drug resistance. Nat Rev Cancer 5: 275-284, 2005.

22. Mani SA, Guo W, Liao MJ, Eaton EN, Ayyanan A, Zhou AY, Brooks M, Reinhard F, Zhang CC, Shipitsin M, et al: The epithelial-mesenchymal transition generates cells with properties of stem cells. Cell 133: 704-715, 2008.

23. Creighton CJ, Li X, Landis M, Dixon JM, Neumeister VM, Sjolund A, Rimm DL, Wong H, Rodriguez A, Herschkowitz JI, et al: Residual breast cancers after conventional therapy display mesenchymal as well as tumor-initiating features. Proc Natl Acad Sci USA 106: 13820-13825, 2009.

24. Chen Y, Shi M, Yu GZ, et al: Interleukin-8, a promising predictor for prognosis of pancreatic cancer. World J Gastroenterol 18: 1123$1129,2012$.

25. Bonnet D and Dick JE: Human acute myeloid leukemia is organized as a hierarchy that originates from a primitive hematopoietic cell. Nat Med 3: 730-737, 1997.

26. Al-Hajj M, Wicha MS, Benito-Hernandez A, Morrison SJ and Clarke MF: Prospective identification of tumorigenic breast cancer cells. Proc Natl Acad Sci USA 100: 3983-3988, 2003.

27. Curley MD, Therrien VA, Cummings CL, Sergent PA, Koulouris CR, Friel AM, Roberts DJ, Seiden MV, Scadden DT, Rueda BR and Foster R: CD133 expression defines a tumor initiating cell population in primary human ovarian cancer. Stem Cells 27: 2875-2883, 2009.

28. Taghizadeh R, Noh M, Huh YH, et al: CXCR6, a newly defined biomarker of tissue-specific stem cell asymmetric self-renewal, identifies more aggressive human melanoma cancer stem cells. PLoS One 5: e15183, 2010 
29. Constantinescu S: Stemness, Fusion and renewal of hematopoietic and embryonic stem cells. J Cell Mol Med 7: 103-112, 2003.

30. Wang SH, Tsai MS, Chiang MF and Li H: A novel NK-type, homebox gene, ENK (early embryo specific NK), Preferentially expressed in embryonic stem cells. Gene ExPr Patterns 3: 99-103, 2003.

31. Mitsui K, Tokuzawa Y, Itoh H, Segawa K, Murakami M, Takahashi K, Maruyama M, Maeda M and Yamanaka S: The homeoprotein Nanog is required for maintenance of pluripotency in mouse epiblast and ES cells. Cell 113: 631-642, 2003.

32. Chambers I, Colby D, Robertson M, Nichols J, Lee S, Tweedie S and Smith A: Functional expression cloning of Nanog, a pluripoteney sustaining factor in embryonic stem cells. Cell 113: 643-655, 2003

33. Brehm A, Ohbo K and Schöler H: The carboxy-terminal transactivation domain of Oct-4 acquires cell specificity through the POU domain. Mol Cell Biol 17: 165-162, 1997.

34. Hart AH, Hartley L, Ibrahim M and Robb L: Identification, cloning and expression analysis of the pluripotency promoting nanog genes in mouse and human. Dev Dyn 230: 187-198, 2004.

35. Boyer LA, Lee TI, Cole MF, Johnstone SE, Levine SS, Zucker JP, Guenther MG, Kumar RM, Murray HL, Jenner RG, et al: Core transcriptional regulatory circuitry in human embryonic stem cells. Cell 122: 947-956, 2005.

36. Jung M, Peterson H, Chavez L, Kahlem P, Lehrach H, Vilo J and Adjaye J: A data integration approach to mapping OCT4 gene regulatory networks operative in embryonic stem cells and embryonal carcinoma cells. PLoS One 5: e10709, 2010.

37. Hoei-Hansen CE, Sehested A, Juhler M, Lau YF, Skakkebaek NE, Laursen $\mathrm{H}$ and Rajpert-de Meyts E: New evidence for the origin of intracranial germ cell tumours from primordial germ cells: Expression of pluripotency and cell differentiation markers. J Pathol 209: 25-33, 2006.

38. Ben-Porath I, Thomson MW, Carey VJ, Ge R, Bell GW, Regev A and Weinberg RA: An embryonic stem cell-like gene expression signature in poorly differentiated aggressive human tumors. Nat Genet 40: 499-507, 2008.

39. Ye F, Zhou C, Cheng Q, Shen J and Chen H: Stem-cell-abundant proteins nanog, nucleostemin and musashil are highly expressed in malignant, cervical epithelial cells. BMC Cancer 8: 108, 2008.

40. Siu MK, Wong ES, Chan HY, Ngan HY, Chan KY and Cheung AN: Overexpression of NANOG in gestational trophoblastic diseases: Effect on, apoptosis, cell invasion and clinical outcome. Am J Pathol 173: 1165-1172, 2008.

41. Chen Z, Xu W, Qian H, et al: Stem cell markers Nanog detected in gastric diagnosis. Clin Exam J 27: 6-8, 2009 (In Chinese)
42. Reiter RE, Gu Z, Watabe T, Thomas G, Szigeti K, Davis E, Wahl M, Nisitani S, Yamashiro J, Le Beau MM, et al: Prostate stem cell antigen: A cell surface marker overexpressed in prostate cancer. Proc Natl Acad Sci USA 95: 1735-1740, 1998.

43. Feng HC, Tsao SW, Ngan HY, Xue WC, Kwan HS, Siu MK, Liao XY, Wong E and Cheung AN: Overexpression of prostate stem cell antigen is associated with gestational trophoblastic neoplasia. Histopathology 52: 167-174, 2008.

44. Ono H, Hiraoka N, Lee YS, Woo SM, Lee WJ, Choi IJ, Saito A, Yanagihara K, Kanai Y, Ohnami S, et al: Prostate stem cell antigen, a presumable organ-dependent tumor suppressor gene, is down-regulated in gallbladder carcinogenesis. Genes Chromosomes Cancer 51: 30-41, 2012.

45. Saeki N, Gu J, Yoshida T and Wu X: Prostate stem cell antigen: A Jekyll and Hyde molecule. Clin Cancer Res 16: 3533-3538, 2010.

46. Study Group of Millennium Genome Project for Cancer; Sakamoto H, Yoshimura K, Saeki N, et al: Genetic variation in PSCA is associated with susceptibility to diffuse-type gastric cancer. Nat Genet 40: 730-740, 2008.

47. Lu Y, Chen J, Ding Y, Jin G, Wu J, Huang H, Deng B, Hua Z, Zhou Y, Shu Y, et al: Genetic variation of PSCA gene is associated with the risk of both diffuse-and intestinal-type gastric cancer in a Chinese population. Int J Cancer 127: 2183-2189, 2010.

48. Qiao L and Feng Y: Genetic variations of prostate stem cell antigen (PSCA) contribute to the risk of gastric cancer for Eastern Asians: A meta-analysis based on 16792 individuals. Gene 493: 83-91, 2012.

49. Lochhead P, Frank B, Hold GL, et al: Genetic variation in the prostate stem cell antigen gene and upper gastrointestinal cancer in white individuals. Gastroenterology 140: 435-441, 2011.

50. Kuroda T, Tada M, Kubota H, Kimura H, Hatano SY, Suemori H, Nakatsuji N and Tada T: Octamer and Sox elements are required. For transcriptional cis regulation of nanog gene expression. Mol Cell Biol 25: 2475-2485, 2005.

51. Brandner S: Nanog, Gli, and p53: A new network of stemness in development and cancer. EMBO J 29: 2475-2476, 2010.

52. Zbinden M, Duquet A, Lorente-Trigos A, Ngwabyt SN, Borges I and Ruiz i Altaba A: NANOG regulates glioma stem cells and is Essential in vivo acting in a cross-functional network with GLI1 and p53. EMBO J 29: 2659-2674, 2010. 